\title{
Pru p 3-Sensitised Italian Peach-Allergic Patients Are Less Likely to Develop Severe Symptoms When Also Presenting IgE Antibodies to Pru p 1 and Pru $p 4$
}

\author{
Elide A. Pastorello ${ }^{a}$ Laura Farioli $^{b}$ Valerio Pravettonid ${ }^{d}$ Joseph Scibilia ${ }^{a}$ \\ Ambra Mascheri $^{a} \quad$ Linda Borgonovo $^{a}$ Marta Piantanidad Laura Primavesi ${ }^{d}$ \\ Chrysi Stafylaraki $^{a}$ Sara Pasqualetti ${ }^{a} \quad$ Jan Schroeder ${ }^{a}$ Michele Nichelattic \\ Alessandro Marocchi ${ }^{\text {b }}$ \\ a Unit of Allergology and Immunology, b Department of Laboratory Medicine, and 'Service of Biostatistics, \\ Niguarda Ca' Granda Hospital, and ' 'Unit of Clinical Allergy and Immunology, Fondazione IRCCS Ca' Granda, \\ Ospedale Maggiore Policlinico, Milan, Italy
}

\section{Key Words}

Peach allergy $\cdot$ Peach allergens $\cdot$ Birch allergy .

Anti-rPru p $1 \cdot$ Anti-rPru p $3 \cdot$ Anti-rPru p $4 \cdot$ Anti-rBet v 1 .

Anti-rBet v $2 \cdot$ Recombinant allergens $\cdot$ Oral allergy

syndrome

\begin{abstract}
Background: The roles played by different peach allergens with respect to symptom severity have not been completely ascertained. We have evaluated the diagnostic efficacy of peach recombinant allergens ImmunoCAP compared to peach in the identification of subjects at an increased risk for severe reactions to peaches. Methods: 148 peach-allergic patients were divided based on their symptom severity into 2 groups: mild oral allergy syndrome (OAS) and severe OAS. Anti-rPru p 1, 3 and 4 lgE levels were measured. Statistical analyses were carried out using parametric and non-parametric tests. Results: anti-rPru $p 1$ and anti-rPru p 4 lgE levels were significantly higher in patients with mild OAS than in
\end{abstract}

patients with severe OAS $(p=0.0001)$; in contrast, anti-rPru $p$ $3 \mathrm{lgE}$ levels were significantly higher in patients with severe OAS than in patients with mild OAS $(p<0.00005)$. Moreover, we found that any unitary increase in anti-rPru $p 1 \mathrm{lgE}$ values corresponded to a $2.48 \%$ reduction in the odds of having severe OAS $(p=0.048)$, whereas any unitary increase in antirPru $p 3 \lg E$ values corresponded to a $9.02 \%$ increase in the probability of having severe OAS $(p=0.001)$. Unexpectedly, we found that patients positive to rPru $\mathrm{p} 3$ as well as rPru $p 1$ and 4 demonstrated a significant reduction of the odds of developing severe symptoms than those positive to rPru $\mathrm{p} 3$ alone. Anti-rPru p 3 lgE levels were a significantly better indicator than anti-peach IgE values $(p=0.016)$ of patients with the highest risk for severe OAS. A cutoff of $2.69 \mathrm{kUA} / \mathrm{l}$ for anti-rPru p 3 IgE values better discriminated peach-allergic patients at a higher risk for symptoms. Conclusions: Italian patients with positive anti-rPru p 1, 4 and 3 IgE levels seemed less likely to experience the clinical effects of high anti-rPru p 3 lgE values.

Copyright $\odot 2011$ S. Karger AG, Basel

\section{KARGER}

Fax +41613061234 E-Mail karger@karger.ch www.karger.com (c) 2011 S. Karger AG, Basel

$1018-2438 / 11 / 1564-0362 \$ 38.00 / 0$

Accessible online at:

www.karger.com/iaa
Correspondence to: Prof. Elide A. Pastorello

Niguarda Ca' Granda Hospital, Unit of Allergology and Immunology

Piazza Ospedale Maggiore 3

IT-20162 Milan (Italy)

Tel. +39 026444 4414, E-Mail allergologia@ ospedaleniguarda.it 


\section{Introduction}

Several epidemiological studies have shown that peach is one of the most important causes of food allergy in adults in Europe [1-6], and that it is the most frequent offending food in Spain [7] and in Italy as demonstrated in a very recent multicenter study [8]. As shown in these studies the most frequent clinical manifestation of peach allergy is the oral allergy syndrome (OAS), however, the literature clearly shows that peach hypersensitivity also can elicit systemic symptoms of variable severity including anaphylaxis [9]. Peach-induced OAS, which is more frequent in Northern than in Southern Europe, is often associated with birch pollen allergy; in these cases, the involved peach allergen is Pru p 1, the Bet $\mathrm{v} 1$ homologue [10]. By contrast, systemic symptoms prevail in Southern Europe, and they seem to be associated with sensitisation to a lipid transfer protein (LTP), namely Pru p 3 [11]. For example, in Spain, Gamboa et al. [12] evaluated sensitisation profiles and clinical symptoms in 30 peach-allergic subjects and showed that 19 patients with systemic symptoms were only sensitised to Pru p 3, whereas 5 patients with mild OAS were only sensitised to peach profilin and/ or to Bet v 1 . However, given the low frequency of birch pollen allergy in Northern Spain, the role of birch pollen exposure in modulating the clinical manifestations of peach allergy could not be ascertained. It is thus important to analyse different clinical manifestations of peach allergy in subjects exposed to birch pollen and predisposed to Pru $\mathrm{p} 3$ sensitisation, as observed in people living in Northern Italy.

The recent commercial availability of the most relevant allergens for peach and birch in recombinant form (rPru p 1, 3, 4 and rBet v 1, 2, 4) allowed us to conduct a prospective study aimed at evaluating the role of these allergens in determining the clinical manifestations of peach allergy. In particular, we tried to identify patients at a high risk for developing severe peach-induced symptoms in a large population of subjects resident in a birchrich area such as Milan by using component-resolved diagnostics for the major peach and birch allergens. At this point we evaluated the relationship between peach allergy severity, defined on the basis of a questionnaire and symptoms score, with anti-rPru p 1, 3, 4 and anti-peach IgE levels. We also evaluated anti-rBet v 1, 2 and 4 specific IgE values to better characterise anti-rPru p 1 and anti-rPru p 4 IgE levels. We took such a broad approach in order to evaluate the role of sensitization to the single peach and birch allergens, but also to study their reciprocal interrelationships.

Sensitisation to Pru p 1, 3 and 4 Gives Less Severe Symptoms than Pru p 3 IgE Alone

\section{Materials and Methods}

Patients

During the period from January to October 2008, all patients referred to the Unit of Allergology and Immunology of the Niguarda Ca' Granda Hospital (Milan, Italy) were consecutively screened for a history of peach allergy. 148 patients, ranging from 13 to 62 years of age, not pregnant and with a clear positive history of immediate-type reaction to peaches, were enrolled in the clinical study after signing the informed consent form.

Independently, two physicians collected all clinical histories using the Case Report Form as part of the standardised protocol. Symptoms were classified into four possible grades of severity, as published elsewhere [13]. Briefly, we defined OAS grade I as the presence of symptoms localised to the oral mucosa. OAS grades II, III and IV were defined as the presence of OAS and systemic symptoms of increasing severity as follows: grade II, OAS + gastrointestinal symptoms (vomiting, diarrhoea and severe abdominal pain); grade III, OAS and/or systemic symptoms (urticaria/ angioedema and/or rhinoconjunctivitis and/or asthma), and grade IV, OAS and/or life threatening symptoms (glottis oedema, hypotension, syncope) [13]. Patients with anaphylaxis after peach ingestion were diagnosed according to Sampson's criteria [14].

Patients were then subdivided into two groups according to symptom severity: mild OAS (group A), OAS grade I only; severe OAS (group B), patients with OAS grades II, III and IV. Patients with mild OAS reintroduced peaches the subsequent spring under our supervision and experienced local symptoms of OAS; patients in the severe OAS group were not challenged because of documented severe peach allergy [15]. The clinical symptoms of peach exposure could not be verified in some group B patients because they had avoided peaches for many years and did not agree to peach reintroduction. Age, gender and the presence of atopy were also evaluated. Ten non-peach-allergic and non-atopic patients were included as negative controls.

\section{Study Design}

This cross-sectional observational study was approved by the Ethics Committee of Niguarda Ca' Granda Hospital and registered at ClinicalTrials.gov, protocol ID NCT00715156. The general objectives of the study were to select patients who were allergic to peaches, and to stratify them into different categories based on symptom severity, checked on the basis of open peach reintroduction with patients diagnosed for having mild OAS and of a clear documentation of the previous reactions for patients with severe OAS. Other objectives were to characterise specific IgE reactivity against peach recombinant allergens (rPru p 1, 3, 4), to evaluate the presence of respiratory symptoms caused by birch pollen, to characterise the specific IgE reactivity against birch recombinant allergens ( $r B e t \mathrm{v} 1,2,4)$ and finally to correlate $\operatorname{IgE}$ reactivity against each individual recombinant allergen to the different clinical symptoms.

For statistical analysis we analyzed the different variables by comparing the patients of Group A to the patients of Group B (OAS II, III and IV).

In vivo Tests

All patients underwent skin prick tests (SPTs) with commercial food and inhalant allergens (1:10 w/v; ALK-Abelló); skin tests with fresh peach were performed using the prick-prick method 
[16]. All patients were also skin tested with purified peach LTP at the concentrations of 2.5, 5, 10 and $20 \mu \mathrm{g} / \mathrm{ml}$. Natural peach LTP was chromatographically purified starting from peach peel extract, as previously described [11]. Skin tests were performed on the volar aspect of the forearm, and histamine dihydrochloride $(10 \mathrm{mg} / \mathrm{ml})$ as well as saline solution were used as positive and negative controls, respectively. The results were evaluated after 15 min, and tests were considered positive if the elicited wheal had a mean diameter of at least $3 \mathrm{~mm}$ [16]. The spectrum of sensitivities to other foods was not included in this study.

\section{In vitro Tests}

Serum levels of anti-rPru p 1, 3 and 4, anti-rBet v 1, 2 and 4, anti-peach and anti-birch IgE antibodies were determined using the ImmunoCAP system according to the manufacturer's instructions. IgE values were considered positive when a value greater than $0.10 \mathrm{kUA} / \mathrm{l}$ was obtained.

\section{Analysis of Subpopulations}

Based on positive (+) or negative (-) specific IgE levels against each recombinant peach allergen, the study population was divided into eight subgroups as follows:

(1) rPru p 3(+), rPru p 1 (-), rPru p 4(-);

(2) rPru p 3(+), rPru p $1(-)$, rPru p 4(+);

(3) rPru p 3(-), rPru p $1(+)$, rPru p 4(-);

(4) rPru p 3(-), rPru p $1(+)$, rPru p 4(+);

(5) rPru p 3(+), rPru p $1(+)$, rPru p 4(-);

(6) rPru p 3(+), rPru p $1(+)$, rPru p 4(+);

(7) rPru p 3(-), rPru p $1(-)$, rPru p 4(+);

(8) rPru p 3(-), rPru p $1(-)$, rPru p 4(-).

Subgroup 1 should be considered the reference population and subgroup 8 contained 10 negative controls, because none of the peach-allergic patients presented negative IgE levels against all three peach recombinant allergens.

\section{Statistical Analysis}

Sample Size

Sample size was calculated from the expected positivity to recombinant rPru p 3 in the considered arms. Assuming that positivity in the group A would have been observed in $40 \%$ of subjects, it has been considered clinically significant to get a positivity at least equal to $65 \%$ in the group B. Using these figures, a two-sided $\chi^{2}$ test with 70 patients per group would have achieved a $81 \%$ power in recognizing a difference of $25 \%$ between groups, with a significance level of 0.05 .

\section{Analysis}

All data were submitted to descriptive analysis: the continuous variables were described by mean and standard deviation, or by median and range, respectively, if the distribution was found normal or non-normal on the basis of the Shapiro-Wilk test. The categorical and binary variables were analysed by cross-tabulation, to obtain the absolute and relative frequencies of their values.

Comparison of categorical variables among groups were carried out with Pearson's $\chi^{2}$ or Fisher's exact test. A generalised linear model with a logit link function was used to assess the dependence of symptom severity on the various peach recombinant allergens. ROC analysis was carried out for each univariate model to determine the optimal cutoff value, if any, for significant continuous independent variables; comparison of ROC curves was carried out according to the DeLong test. Spearman's rank coefficient $(\rho)$ was used to quantify the correlation between all variables, both in vivo and in vitro. The comparison of continuous variables was carried out with the Student t test in case of normal distribution, and with the Mann-Whitney $U$ test (two groups) or Kruskal-Wallis test (more than two groups) in case of non-normal distribution. Statistical significance was assumed at $p<0.05$. Data analysis was carried out using Stata/SE 11.1 (Stata Corp Inc.).

\section{Results}

\section{Patients}

148 peach-allergic subjects were selected, comprising 107 females and 41 males $(\mathrm{p}<0.001)$ with a median age of 37 years (range 13-62), no significant age differences between genders ( $p=0.951)$. Of these patients, 76 were classified as mild OAS (group A) and 72 as severe OAS (group B). In the latter group, 14 patients (19.5\%) were classified as OAS grade II, 49 (68\%) as grade III and 9 $(12.5 \%)$ as grade IV, that is, life-threatening anaphylaxis. No differences were observed in OAS severity with respect to gender $(\mathrm{p}=0.703)$ or age $(\mathrm{p}=0.759)$.

All patients had positive SPTs for fresh peach. We observed that $50 / 148$ patients $(33.8 \%)$ had a positive SPT for peach LTP at a concentration of $2.5 \mu \mathrm{g} / \mathrm{ml}, 19 / 148$ (12.8\%) at a concentration of $5 \mu \mathrm{g} / \mathrm{ml}, 12 / 148(8.1 \%)$ at a concentration of $10 \mu \mathrm{g} / \mathrm{ml}$ and $7 / 148(4.7 \%)$ at a concentration of $20 \mu \mathrm{g} / \mathrm{ml}$. It is noteworthy that skin reactivity to purified peach LTP was significantly positively related to antirPru p 3 IgE values (Spearman's test $\rho=0.574$; $p<0.0005$ ) and negatively related to anti-rPru p $1 \mathrm{IgE}$ values (Spearman's test $\rho=-0.363$; $\mathrm{p}<0.0005)$.

We observed skin positivity to birch pollen in $81 / 148$ patients (54.7\%), among whom 63 patients (77.8\%) also presented with birch pollen symptoms. Table 1 shows the significant differences between group A and group B patients with respect to pollen sensitisation and pollen symptoms. Respiratory symptoms were significantly more frequent in the mild OAS group; no differences were observed in the frequency of asthma between the two groups (28 patients in the mild OAS group and 30 in the severe OAS group, data not shown).

\section{Descriptive Results (ImmunoCAP Results for \\ Anti-Peach and Anti-Birch Specific IgE and Anti-rPru \\ p 1, 3 and 4 and Anti-rBet v 1, 2 and 4 Specific IgE) in Patients with Mild and Severe OAS \\ All 148 patients presented positive peach Immuno-} CAP results (IgE value $>0.10 \mathrm{kUA} / \mathrm{l}$ ), whereas 106 patients had positive IgE levels against birch ImmunoCAP. 
Table 1. Comparison by Fisher's exact test of mild and severe OAS groups as regards pollen and specific IgE ImmunoCAP values

\begin{tabular}{llll}
\hline Inhalant allergen & Mild OAS & Severe OAS & p values \\
\hline Birch & & & \\
$\quad$ ImmunoCAP & $65(85.50 \%)$ & $37(51.40 \%)$ & $<0.0005$ \\
$\quad$ Symptoms & $45(59.20 \%)$ & $18(2.50 \%)$ & $<0.0005$ \\
Ragweed & & & \\
$\quad$ ImmunoCAP & $59(77.60 \%)$ & $41(56.90 \%)$ & 0.009 \\
$\quad$ Symptoms & $20(26.30 \%)$ & $14(19.40 \%)$ & 0.336 \\
Grass & & & \\
$\quad$ ImmunoCAP & $55(72.30 \%)$ & $28(38.80 \%)$ & $<0.0005$ \\
$\quad$ Symptoms & $42(55.20 \%)$ & $26(36.10 \%)$ & 0.022 \\
Mugwort & & & \\
$\quad$ ImmunoCAP & $54(71 \%)$ & $58(80.50 \%)$ & 0.187 \\
$\quad$ Symptoms & $15(19.70 \%)$ & $9(12.50 \%)$ & 0.271 \\
Wall pellitory & & & \\
$\quad$ ImmunoCAP & $51(67.10 \%)$ & $35(48.60 \%)$ & 0.030 \\
$\quad$ Symptoms & $10(13.10 \%)$ & $3(4.10 \%)$ & 0.080 \\
\hline
\end{tabular}

A greater percentage of positive ImmunoCAP values and symptoms for all of the considered pollens were detected in the mild OAS group, with the exception of mugwort which was high in both groups even if group B showed slightly more sensitisation with respect to group $\mathrm{A}$, even if symptoms were lower in group $\mathrm{B}$ patients.

Table 2 shows the number and percentage of patients with different sensitisation profiles to rPru p 1, 3 and 4 and rBet $v 1,2$ and 4 in the two groups. In the group of patients with mild OAS, 50/76 patients (65.8\%) were sensitised to rPru p 1, 41/76 patients (53.9\%) to rPru p 3 and $31 / 76$ patients $(40.8 \%)$ to $\mathrm{rPru} \mathrm{p} 4$. In the group of patients with severe OAS, 23/72 patients (31.9\%) were sensitised to $\mathrm{rPru}$ p 1,63/72 patients (87.5\%) to rPru p 3 and $13 / 72$ patients (18.1\%) to rPru $\mathrm{p} 4$. In the group of patients with severe OAS, the number of patients who were monosensitised to rPru $\mathrm{p} 3$ was six times greater than the number of patients who were monosensitised to rPru $\mathrm{p} 1$. Thirty-four of 104 patients (32.7\%) sensitised to rPru p 3 were also sensitised to $\mathrm{rPru} \mathrm{p} 1$.

For birch recombinant allergens, all patients in the group with mild OAS presented positive IgE levels to at least one birch recombinant allergen, in comparison to $30 / 72(41.6 \%)$ patients in the group with severe OAS. In the group with mild OAS, $62 / 76$ patients $(81.6 \%)$ were sensitised to $\mathrm{rBet} \mathrm{v} 1,44 / 76$ patients (57.9\%) to rBet $\mathrm{v} 2$ and $24 / 76$ patients (31.6\%) to rBet $\mathrm{v} 4$. In the group with severe OAS, 26/72 patients (36.1\%) were sensitised to rBet $\mathrm{v} 1$, $13 / 72$ patients (18.1\%) to rBet $\mathrm{v} 2$ and $3 / 72$ patients (4.2\%) to rBet $\mathrm{v} 4$.

Sensitisation to Pru p 1, 3 and 4 Gives Less Severe Symptoms than Pru p 3 IgE Alone
Table 2. Patients with mild (76) and severe (72) OAS demonstrating positive IgE values for peach and birch recombinant allergens, both alone and in combination

\begin{tabular}{lllll}
\hline Positive IgE values & $\begin{array}{l}\text { Mild OAS } \\
(\mathrm{n}=76)\end{array}$ & $\begin{array}{l}\text { Severe OAS } \\
(\mathrm{n}=72)\end{array}$ & $\begin{array}{l}\text { Total } \\
(\mathrm{n}=148)\end{array}$ \\
\cline { 2 - 3 } & $\frac{\mathrm{n} \%}{\mathrm{n}} \%$ & & $\mathrm{n} \%$ \\
\hline
\end{tabular}

\section{Peach}

$\begin{array}{lllllll}\text { anti-rPru p 1 } & 20 & 13.5 & 7 & 4.7 & 27 & 18.2\end{array}$ $\begin{array}{lllllll}\text { anti-rPru p 3 } & 19 & 12.8 & 44 & 29.7 & 63 & 42.5\end{array}$ $\begin{array}{lllllll}\text { anti-rPru p } 4 & 5 & 3 & 0 & 0 & 5 & 3\end{array}$ $\begin{array}{lrlllll}\text { anti-rPru p 1-3-4 } & 14 & 9.4 & 6 & 4 & 20 & 13.5\end{array}$ $\begin{array}{lllllll}\text { anti-rPru p 1-3 } & 6 & 4 & 8 & 5.4 & 14 & 9.4\end{array}$ $\begin{array}{lllllll}\text { anti-rPru p 1-4 } & 10 & 6.7 & 2 & 1.3 & 12 & 8.1\end{array}$

\begin{tabular}{rrrrrrr} 
anti-rPru p 3-4 & 2 & 1.3 & 5 & 5 & 7 & 4.7 \\
\hline
\end{tabular}

\begin{tabular}{lllllll}
\hline Total & 76 & 100 & 72 & 100 & 148 & 100
\end{tabular}

\begin{tabular}{lllll}
\hline Total anti-rPru p 1 & 50 & 65.8 & 23 & 31.9
\end{tabular}

$\begin{array}{lllll}\text { Total anti-rPru p } 3 & 41 & 53.9 & 63 & 87.5\end{array}$

$\begin{array}{lllll}\text { Total anti-rPru p } 4 & 31 & 40.8 & 13 & 18.1\end{array}$

\begin{tabular}{lrrrrrr}
\hline Birch & & & & & & \\
anti-rBet v 1 & 23 & 15.5 & 16 & 10.8 & 39 & 26.3 \\
anti-rBet v 2 & 5 & 3.4 & 3 & 2.0 & 8 & 5.4 \\
anti-rBet v 4 & 1 & 0.7 & 0 & 0 & 1 & 0.7 \\
anti-rBet v 1-2-4 & 7 & 4.7 & 1 & 0.7 & 8 & 5.4 \\
anti-rBet v 1-2 & 24 & 16.2 & 8 & 5.4 & 32 & 21.6 \\
anti-rBet v 1-4 & 8 & 5.4 & 1 & 0.7 & 9 & 6.1 \\
anti-rBet v 2-4 & 8 & 5.4 & 1 & 0.7 & 9 & 6.1 \\
\hline Total & 76 & 100 & 30 & 41.6 & 106 & 71.6 \\
\hline Total anti-rBet v 1 & 62 & 81.6 & 26 & 36.1 & & \\
Total anti-rBet v 2 & 44 & 57.9 & 13 & 18.1 & & \\
Total anti-rBet v 4 & 24 & 31.6 & 3 & 4.2 & &
\end{tabular}

A clear prevalence of Pru p 3 sensitisation in the severe OAS group and a slight predominance of Bet $\mathrm{v} 1$ sensitisation in the mild OAS group were detected.

\section{Univariate Analysis (to Compare Variables Using \\ Parametric and Non-Parametric Tests)}

Figure 1a shows that anti-rPru p 1 IgE levels were significantly higher in patients with mild OAS than in patients with severe OAS ( $\mathrm{p}=0.0001)$; in contrast, anti-rPru p 3 IgE levels (fig. $1 b$ ) were significantly higher in patients with severe OAS than in patients with mild OAS $(\mathrm{p}<0.00005)$. We also observed that anti-rPru $\mathrm{p} 4 \operatorname{IgE}$ levels were significantly higher in the mild OAS group when compared to the severe OAS group $(\mathrm{p}=0.0017)$ (fig. 1c).

Next, we evaluated the relationship between anti-rPru p 1 and anti-rPru p 3 IgE levels and discovered a negative correlation (Spearman's test $\rho=-0.4920 ; p<0.00005$ ), 

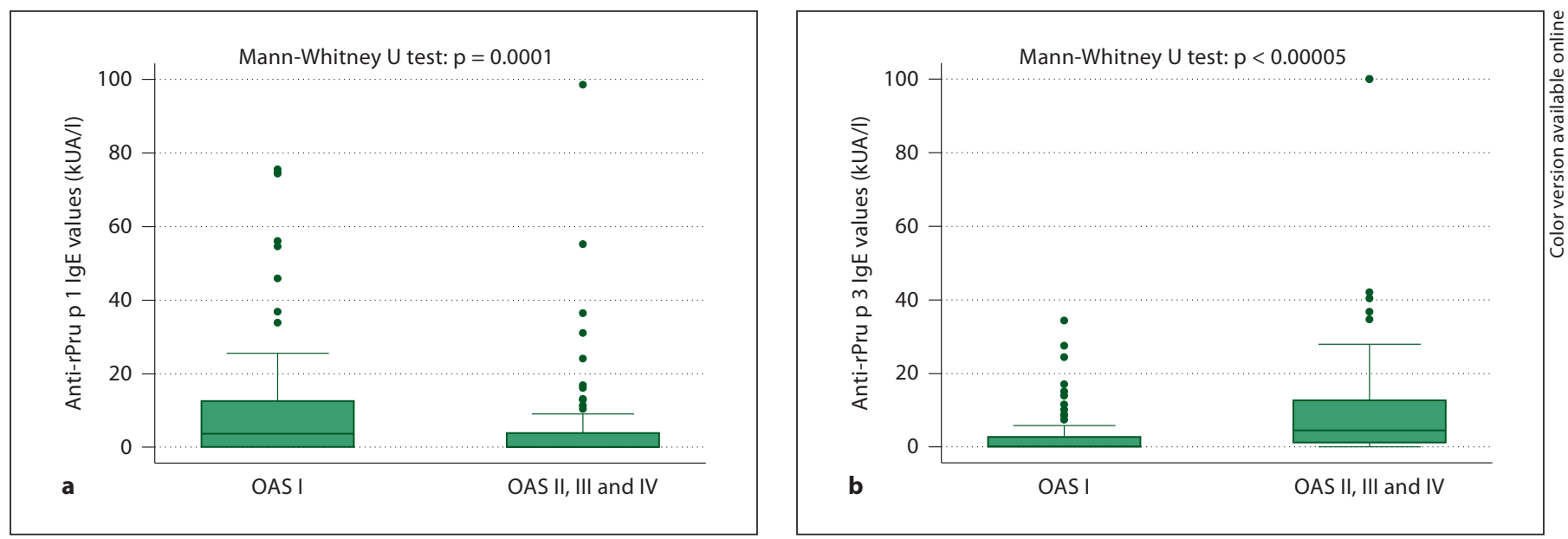

Fig. 1. Comparison of anti-rPru p 1 (a), anti-rPru p 3 (b) and antirPru p 4 (c) IgE values in mild and severe OAS patients. The results are expressed as kUA/l.

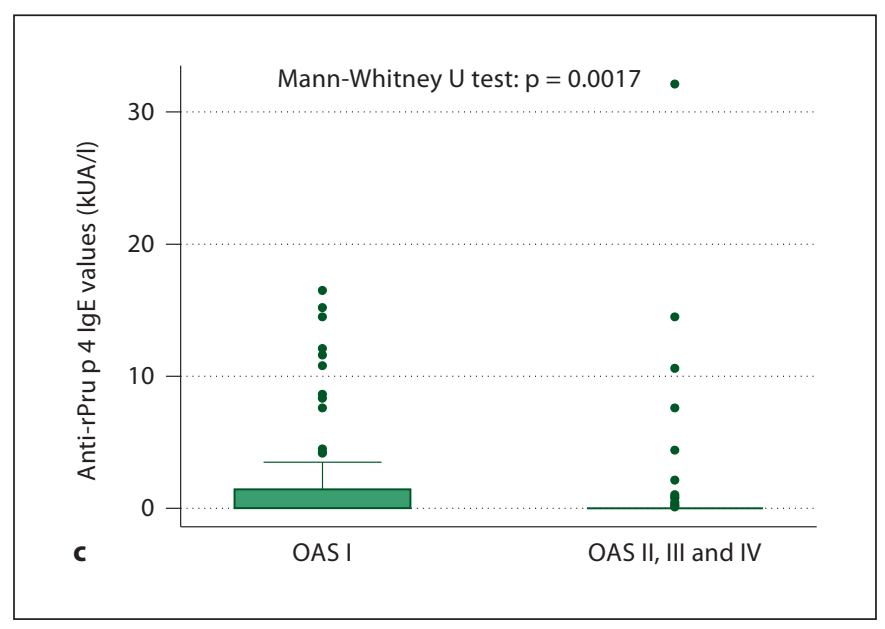

that is, for each single patient we found that the higher the titre for anti-rPru p 1 IgE the lower the titre for anti-rPru p 3 IgE. Furthermore, the generalised linear model revealed a significant association between both anti-rPru $p$ 1 and anti-rPru p $3 \mathrm{IgE}$ values and symptom severity. More precisely, any arbitrary unitary increase $(+1 \mathrm{kUA} / \mathrm{l})$ in anti-rPru p $1 \mathrm{IgE}$ values corresponded to a $2.48 \%$ reduction (95\% CI $0.02-4.89 \%)$ in the odds of developing severe OAS $(\mathrm{p}=0.048)$, and any arbitrary unitary increase $(+1 \mathrm{kUA} / \mathrm{l})$ in anti-rPru p 3 IgE values corresponded to a $9.02 \%$ increase $(95 \%$ CI $3.60-14.72 \%)$ in the odds of developing severe OAS ( $\mathrm{p}=0.001)$. Only anti-rPru $\mathrm{p} 1$ and anti-rPru $\mathrm{p} 3 \mathrm{IgE}$ values were significantly associated with OAS severity, whereas anti-rPru p 4 IgE levels were $\operatorname{not}(\mathrm{p}=0.208)$.

Anti-rPru 3 IgE Cutoff Values

When considering all of our patients, if we take the ones with anti-rPru p 3 IgE levels $>0$ and those with anti-rPru $\mathrm{p} 3 \mathrm{IgE}=0$, the odds ratio of a severe reaction are 5.96 (95\% CI 2.44-14.65\%).

We further analysed the sensitivity (SE) and specificity (SP) of anti-rPru $\mathrm{p} 1$ and anti-rPru p $3 \mathrm{IgE}$ values using ROC curves. Reliable cutoff values were not produced for anti-rPru p 1 IgE levels; thus, we focused our attention only on anti-rPru $\mathrm{p} 3 \mathrm{IgE}$ values. The maximum accuracy achieved by the model was $70 \%$, which was observed at two different anti-rPru p 3 IgE cutoff values: $0.39 \mathrm{kUA} / \mathrm{l}$ with $84.7 \%$ SE and $55.3 \%$ SP; $2.28 \mathrm{kUA} / 1$ with $68.1 \% \mathrm{SE}$ and $71.1 \%$ SP; the third cutoff of $2.69 \mathrm{kUA} / \mathrm{l}$, with $63.9 \%$ SE and $75.0 \%$ SP, showed an accuracy of $69 \%$. Given these data, an anti-rPru p 3 IgE value of $2.69 \mathrm{kUA} / \mathrm{l}$ appeared to better discriminate peach-allergic patients at risk for developing more severe symptoms.

Pastorello et al. 

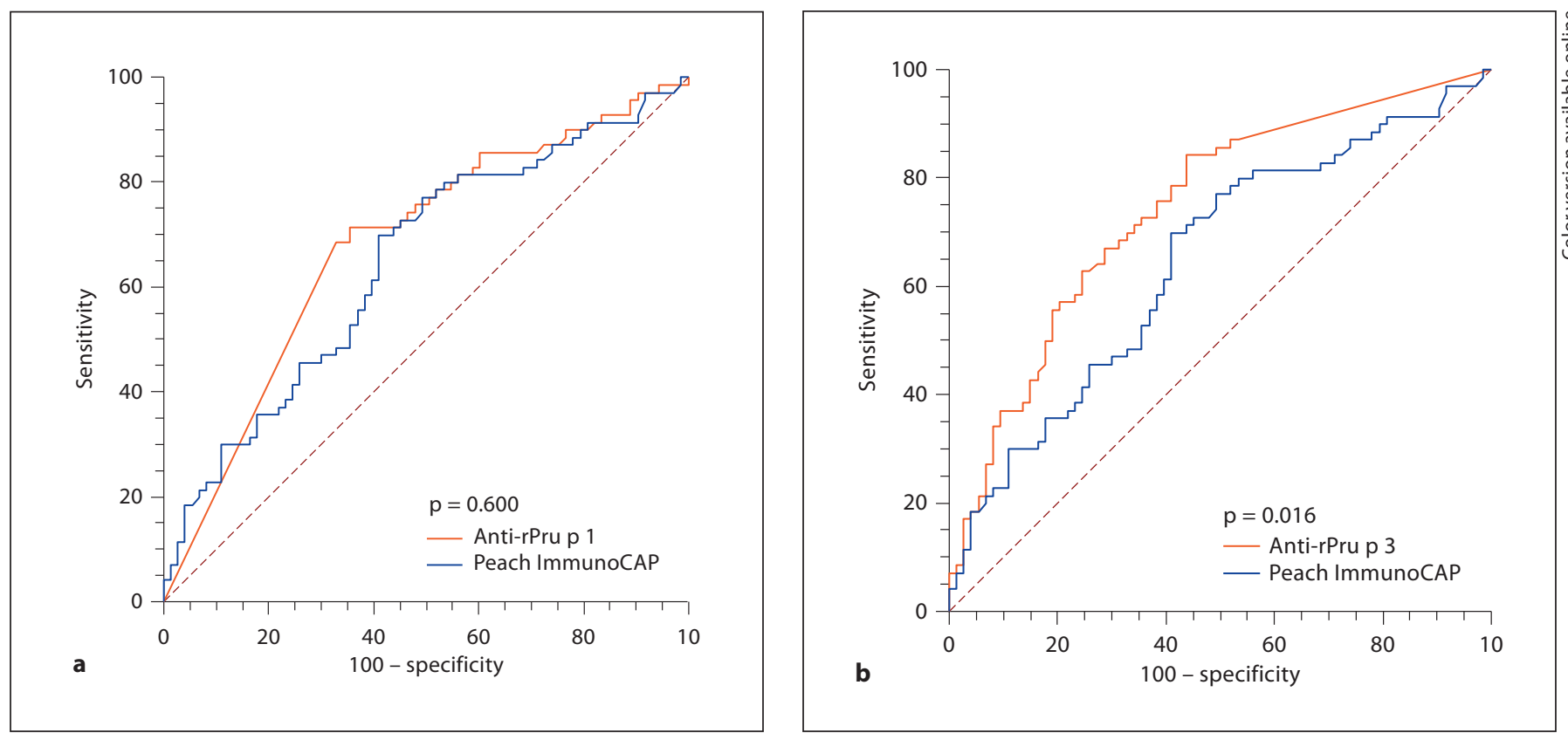

Fig. 2. ROC curves for anti-rPru p 1 and anti-peach IgE values (a) as well as for anti-rPru p 3 and anti-peach IgE values (b). The curves show that anti-rPru p 3 IgE values predict severe reactions significantly better than do anti-peach IgE values.
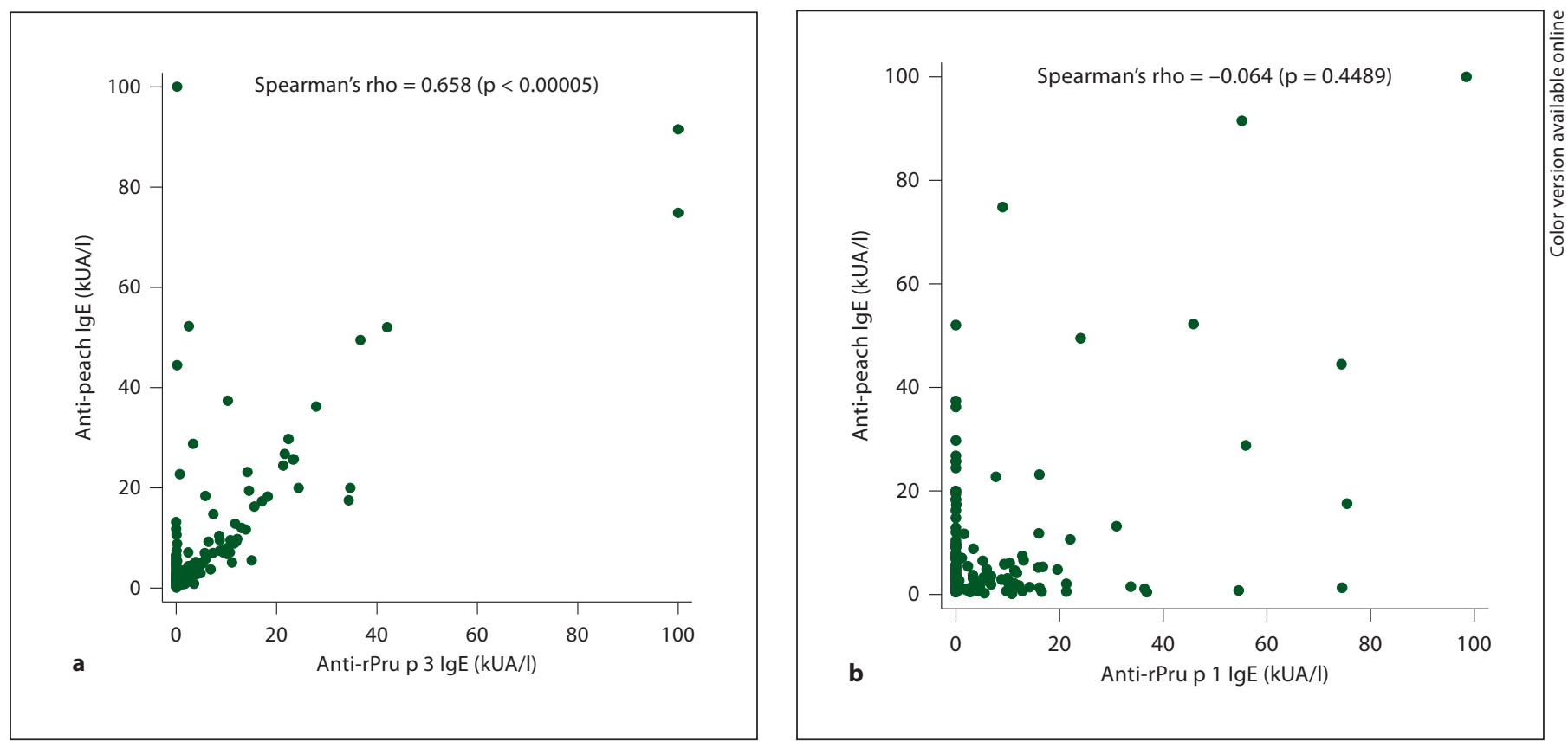

Fig. 3. Correlation between anti-rPru p 3 and anti-peach IgE values (a) and anti-rPru p 1 IgE and anti-peach IgE values (b). 

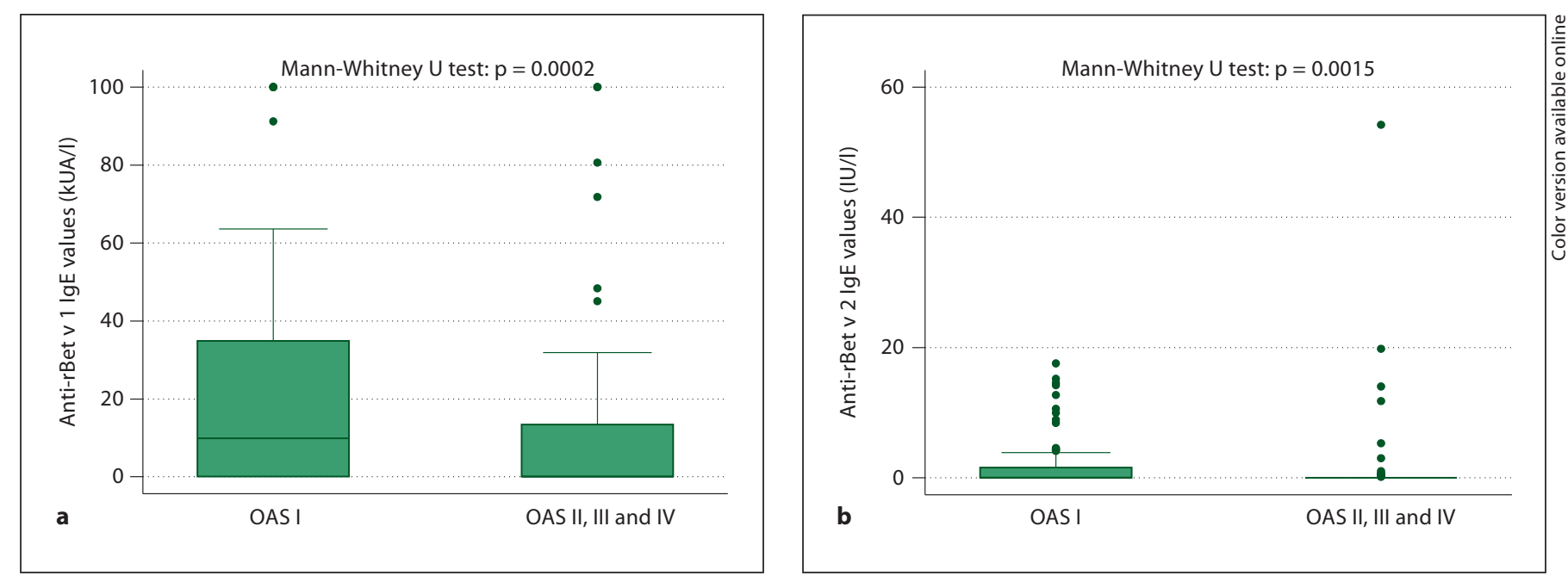

Fig. 4. Comparison of anti-rBet v 1 (a) and anti-rBet v 2 (b) IgE levels in patients with mild and severe OAS.

Comparison of the Diagnostic Performance of

Anti-Peach, Anti-rPrup 1 and Anti-rPrup 3 IgE

The SE and SP of anti-rPru p 1 and anti-rPru p 3 IgE values in comparison with anti-peach $\operatorname{IgE}$ values were evaluated using ROC curves. Figure 2a shows that there was no significant association between anti-peach $\operatorname{IgE}$ values and anti-rPru $p 1 \operatorname{IgE}$ values $(p=0.600)$. Figure $2 b$ shows that anti-rPru p 3 IgE levels were significantly more sensitive than anti-peach $\operatorname{IgE}$ values $(\mathrm{p}=0.016)$ in discriminating peach-allergic patients at risk for developing severe OAS.

\section{Correlation between Anti-Peach and Anti-rPrup 3 and Anti-rPrup 1 IgE Values}

Anti-peach IgE values were significantly related to anti-rPru p 3 IgE values (Spearman's test $\rho=0.658$; p < $0.0005)$ (fig. 3a), whereas no correlation with anti-rPru $p$ $1 \mathrm{IgE}$ values was detected (Spearman's test $\rho=-0.064$; $\mathrm{p}=0.4489$ ) (fig. 3b).

\section{The Relationship between OAS Severity and Anti-rBet} $v$ 1, 2 and 4 IgE Levels

Anti-rBet v 1 IgE levels were significantly higher in the mild OAS group compared to the severe OAS group ( $\mathrm{p}=$ 0.0002) (fig. 4a), and using the generalised linear model, we observed a significant association between the measured anti-rBet v 1 IgE values and OAS severity (Wald's test: $\mathrm{p}=0.018)$. In fact, a unitary increase in anti-rBet $\mathrm{v} 1$ $\mathrm{IgE}$ values corresponded to a $1.83 \%$ reduction in the odds of developing severe OAS (95\% CI 3.31-0.32\%).
Anti-rBet v 2 IgE values were also higher in the mild OAS group than in the severe OAS group $(p=0.0015)$ (fig. 4b). However, using the generalised linear model, no significant associations were found between anti-rBet $\mathrm{v} 2$ IgE values and OAS severity (Wald's test: $\mathrm{p}=0.565$ ).

Similarly to what has been shown by other authors in apple [17], we found a correlation between anti-rPru p 1 and anti-rBet v 1 IgE values (Spearman's test $\rho=0.9717$; $\mathrm{p}<0.00005)$, as well as between anti-rPru p 4 and antirBet 2 IgE levels (Spearman's test $\rho=0.9836 ; \mathrm{p}<0.00005$ ), revealing that sensitisation to Pru p 1 and Pru p 4 depends on sensitisation to Bet v1 and Bet v 2, respectively.

\section{Analysis of Subpopulations}

In the whole study population the values of the 6 recombinant allergens (Bet v 1, Bet v 2, Bet v 4; Pru p 1, Pru p 3, Pru p 4) that we measured for each patients showed a great number of interrelationships among them (table 3). Therefore, we investigated the odds of developing severe reactions in seven subpopulations, we divided the subpopulations on the basis of positive or negative $\operatorname{IgE}$ values for rPru p 1, rPru 3 and rPru p 4. The eighth subgroup, with negative IgE values for all three peach recombinant allergens, consisted of the negative control patients.

We used the general linear model with the subpopulations as a categorical independent variable [where the condition anti-rPru p 3(+)/anti-rPru p 1(-)/anti-rPru p 4(-) IgE values was used as a reference] and symptom severity as a binary dependent variable, to determine the 
Table 3. Spearman's rank correlation values between anti-rBet v 1, anti-rBet v 2, anti-rBet v 4, anti-rPru p 1, anti-rPru p 3 and anti-rPru p 4 IgE values in all 148 patients

\begin{tabular}{|c|c|c|c|c|c|}
\hline & anti-rBet v 1 & anti-rBet v 2 & anti-rBet v 4 & anti-rPru p 1 & anti-rPru p 3 \\
\hline anti-rBet v 2 & $\begin{array}{l}0.3660 \\
0.0001\end{array}$ & & & & \\
\hline anti-rBet v 4 & $\begin{array}{l}0.2378 \\
0.0541\end{array}$ & $\begin{array}{r}0.3975 \\
<0.0001\end{array}$ & & & \\
\hline anti-rPru p 1 & $\begin{array}{r}0.9716 \\
<0.0001\end{array}$ & $\begin{array}{l}0.3454 \\
0.0003\end{array}$ & $\begin{array}{l}0.2473 \\
0.0366\end{array}$ & & \\
\hline anti-rPru p 3 & $\begin{array}{l}-0.4920 \\
<0.0001\end{array}$ & $\begin{array}{r}-0.1614 \\
0.7633\end{array}$ & $\begin{array}{l}-0.1224 \\
>0.9999\end{array}$ & $\begin{array}{l}-0.4950 \\
<0.0001\end{array}$ & \\
\hline anti-rPru p 4 & $\begin{array}{l}0.3685 \\
0.0001\end{array}$ & $\begin{array}{r}0.9836 \\
<0.0001\end{array}$ & $\begin{array}{r}0.3898 \\
<0.0001\end{array}$ & $\begin{array}{l}0.3519 \\
0.0002\end{array}$ & $\begin{array}{r}-0.1579 \\
0.8283\end{array}$ \\
\hline
\end{tabular}

A negative coefficient indicates an inverse correlation. The second row shows the significance ( $\mathrm{p}$ value) of the coefficient shown directly above; all p values were adjusted according to the Bonferroni method for multiple comparisons.

Table 4. Odds ratio of severe reactions for all subpopulations identified, using the subpopulation with anti-rPru p 3 positive IgE values only as a reference

\begin{tabular}{|c|c|c|c|c|c|}
\hline \multirow[t]{2}{*}{ No. } & \multirow[t]{2}{*}{ Subpopulation } & \multirow[t]{2}{*}{ Patients } & \multicolumn{3}{|c|}{$\begin{array}{l}\text { Compared to anti-rPru p 3(+) anti-rPru p 1(-) } \\
\text { anti-rPru p } 4(-)(\mathrm{n}=63)\end{array}$} \\
\hline & & & odds ratio & $95 \% \mathrm{CI}$ & $\mathrm{p}$ values \\
\hline$(2)$ & anti-rPru p 3(+) anti-rPru p 1(-) anti-rPru p 4(+) & 7 & 1.0795 & $0.1921-6.0640$ & 0.931 \\
\hline (3) & anti-rPru p 3(-) anti-rPru p 1(+) anti-rPru p 4(-) & 27 & 0.1511 & $0.0548-0.4171$ & $<0.0005$ \\
\hline (4) & anti-rPru p 3(-) anti-rPru p 1(+) anti-rPru p 4(+) & 12 & 0.0863 & $0.1725-0.4323$ & 0.003 \\
\hline$(5)$ & anti-rPru p 3(+) anti-rPru p 1(+) anti-rPru p 4(-) & 14 & 0.5758 & $0.1756-1.8876$ & 0.362 \\
\hline$(6)$ & anti-rPru p 3(+) anti-rPru p 1(+) anti-rPru p 4(+) & 20 & 0.1851 & $0.0618-0.5549$ & 0.003 \\
\hline$(7)$ & anti-rPru p 3(-) anti-rPru p 1(-) anti-rPru p 4(+) & 5 & n.d. & n.d. & n.d. \\
\hline
\end{tabular}

An odds ratio greater than one indicates an increased risk for a severe reaction. n.d. = Not determined due to a small number of patients.

odds ratio of developing a severe reaction with respect to the reference subpopulation (table 4).

In this model the presence of only positive anti-rPru $\mathrm{p}$ 1 IgE antibodies [anti-rPru p 3(-)/anti-rPru p 1(+)/antirPru p 4(-) subpopulation] indicates a statistically significant reduction $(\mathrm{p}<0.0005)$ in the odds of developing severe symptoms with respect to the reference subpopulation, that is, anti-rPru p 3(+)/anti-rPru p 1(-)/anti-rPru p 4(-). However, when both anti-rPru p 1 IgE and antirPru p 3 IgE values are simultaneously positive in a subpopulation, there is no significant improvement in the odds of developing a severe reaction with respect to the reference population if the anti-rPru p $4 \mathrm{IgE}$ levels are negative (subpopulation 5). This case is clearly evident in subpopulation 6 , where a significant reduction $(\mathrm{p}=0.003)$ in the odds of developing severe symptoms with respect to the reference population [that is, anti-rPru p 3(+)/antirPru $\mathrm{p}$ 1(-)/anti-rPru $\mathrm{p} 4(-)$ ] is only observed in anti-rPru p 3(+)/anti-rPru p 1(+)/anti-rPru p 4(+) patients (subpopulation 6). The subpopulation of anti-rPru $\mathrm{p} 3(-)$ /antirPru p 1(-)/anti-rPru p 4(+) patients was too poorly represented (only 5 patients) to allow a statistical analysis. 


\section{Discussion}

In this study, we selected 148 Italian patients with a food allergy to peaches; we assessed the severity of their reactions using an OAS severity score and evaluated the in vitro $\operatorname{IgE}$ reactivity to peach recombinant allergens rPru p 1, 3 and 4 as well as to rBet v 1,2 and 4 using the ImmunoCAP system.

All patients were assigned to one of two groups according to OAS severity: mild OAS (mucosal symptoms, controlled by peach challenge, group A) and severe OAS (mucosal symptoms as well as systemic reactions of variable severity, closely documented, group B). We found a statistically significant association between mild OAS and anti-rPru p 1 IgE levels, whereas severe OAS was significantly associated with higher anti-rPru $\mathrm{p} 3 \mathrm{IgE}$ levels. Given this observation, we attempted to correlate the two variables (anti-rPru p 1 and anti-rPru p 3) and found a significant inverse Spearman's rank correlation ( $\mathrm{p}<$ $0.0005)$ that led us to hypothesise that elevated anti-rPru $\mathrm{p} 1 \mathrm{IgE}$ values are less likely to be associated to sensitisation to Pru p 3.

Moreover, in this large population of peach-allergic patients living in Northern Italy and exposed to birch pollen, we found that sensitisation to Pru p 1 and Pru p 3 could coexist in a single patient, however, the two sensitisations had different clinical connotations. At present this scenario of co-sensitization seems to be unique to Northern Italy inasmuch as Bet $\mathrm{v} 1$ has been shown to be lacking in Southern Europe and Pru p 3 sensitisation does not occur in Northern Europe due to unclear reasons. We thus focused our attention on this unusual population of patients, allowing us to verify the clinical response through 'field observation' on a large number of peachallergic subjects.

Because the majority of anti-rPru p 1(+) patients also demonstrated positive anti-rPru p 4 IgE values, we decided to explore the likelihood of developing severe reactions in eight subpopulations, determined by the permutation of positive or negative values of anti-rPru $\mathrm{p} 1$, antirPru p 3 and anti-rPru p 4 IgE antibodies, to better understand the clinical outcome of sensitisation to all allergens. We observed evidence that co-sensitised patients with positive anti-rPru p 1 IgE levels had a reduced likelihood of Pru p 3-induced severe symptoms. However, this effect was not significant in the subpopulation of patients positive to Pru p 3 and Pru p 1 but negative to Pru p 4 (subgroup 5), this was most likely due to the small size of this group (only 14 patients; table 4 ). In fact, when we considered the entire population the presence of positive an-
ti-rPru p 1 IgE values in the univariate analysis (73 patients) resulted in a reduction in the likelihood of developing severe symptoms by about $80 \%(p<0.0005)$ as compared to the presence of positive anti-rPru p $3 \operatorname{IgE}$ levels. Therefore, when we analysed the subpopulations, the presence of anti-rPru p 1 and anti-rPru p 4 IgE levels, in anti-rPru p 3(+) patients, seemed to enhance the 'protective' role of positive anti-rPru $\mathrm{p} 1 \mathrm{IgE}$ values on symptom severity ( $p=0.003)$, inasmuch as the simultaneous presence of both antibodies (that is, anti-rPru p 1 and 4) reduced the risk of severe reactions by about $90 \%$. However, the subpopulation that was positive to rPru p 4 but negative to rPru $\mathrm{p} 3$ and rPru $\mathrm{p} 1$ (subgroup 7; table 4) was too small $(n=5)$ to allow for the detection of any Pru $p$ 4 -induced effect versus the reference subgroup. These results can be interpreted in the light of the different geographic distribution of sensitisation and clinical symptoms to peaches and other fruits across Europe. In fact, allergic reactions are more severe and more frequently associated with LTP (for example Pru p 3) sensitisation in Southern Europe; conversely, in Northern Europe, symptoms caused by the same fruit are generally mild and induced by Bet v 1 homologues in fruit [17]. In Spain, Fernandez-Rivas et al. [17] used an apple allergy model to show that a severe allergy to apple is related to Mal d 3 sensitisation, with an odds ratio of 7.76 (95\% CI 3.87$15.56 \%)$ for the development of systemic reactions. This result is similar to our finding of an odds ratio of 5.96 (95\% CI 2.44-14.65\%) for peach-induced severe systemic reactions when anti-rPru 3 IgE antibodies are $>0$, in our entire population. Fernandez-Rivas et al. [17] also found that sensitisation to Bet $\mathrm{v} 1$ had a protective effect against Mal d 3 sensitisation (decrease in odd ratio of 0.29 ). We confirmed analogous results for Pru $\mathrm{p} 3$ and Bet $\mathrm{v} 1$. However, our original finding was that peach-allergic subjects sensitised to Bet $\mathrm{v} 1$ and Bet $\mathrm{v} 2$ homologues might also be sensitised to Pru p 3, and that subjects who had positive anti-Pru p 1, anti-Pru p 4 and anti-Pru p 3 IgE levels had a reduced likelihood of severe symptoms with respect to patients positive to only anti-rPrup 3 . Therefore, it appears that the presence of anti-rPru $\mathrm{p} 1$ and anti-rPru $\mathrm{p} 4$ IgE antibodies actually counteracted the effects of antirPru p 3 IgE antibodies. Although this clinical observation was statistically very significant, it is difficult to explain the grounds for this evidence, thus we can only try and forward some speculative hypotheses. For example, if one considers the rather similar molecular weights of the three allergens, one can suppose that anti-rPru $\mathrm{p} 1$ and anti-rPru p 4 IgE antibodies may somehow impede the facilitated access of anti-rPru p 3 IgE to its allergen on 
grounds of spatial antagonism and not on grounds of immunological competition. On the other hand, given the elevated water-soluble nature of Bet $\mathrm{v} 1$ homologues, such as Pru p 1 and Pru p 4, it is possible to conceive that these proteins immediately react with mast cells in the oral mucosa, thus causing OAS and the release of mast cell-derived serine protease such as tryptase in the local environment, and this protein could partially digest Pru $\mathrm{p} 3$ acting in a trypsin-like manner. Tryptase is a trypsin-like serine protease with a cleavage site that has been shown to contain arginine [18], and recently some investigators have shown that threonine-39 and arginine-40 are the only active cleavage site of trypsin in Pru p 3 [19]. Thus, it is possible that the tryptase delivered by mast cell degranulation secondary to Pru 1 exposure can cleave and thus partially degrade Pru $\mathrm{p} 3$ at the arginine- 40 site, the latter being contained in one of the principal IgE epitopes of Pru p 3.

On the contrary, the different clinical manifestations cannot be attributed to lower levels of anti-rPru p $3 \mathrm{IgE}$ values in the subpopulation of patients positive to Pru $p$ 3, Pru p 1 and Pru p 4, with respect to those only positive to Pru $\mathrm{p} 3$, given that this group unexpectedly presented lower levels of anti-rPru p 3 IgE values.

Another important result of this study was the determination of a cutoff level for anti-rPru $\mathrm{p} 3 \mathrm{IgE}$ values that can identify patients at greater risk for developing severe allergic reactions to peach that was determined by ROC curves. We found that an anti-rPru p 3 IgE value of 2.69 $\mathrm{kUA} / \mathrm{l}$ had the best SE (63.9\%) and SP (75\%) to better discriminate peach-allergic patients at risk for developing more severe symptoms. It is interesting to observe that the anti-peach IgE levels were significantly related (Spearman's test $\rho=0.658 ; p<0.0005)$ to anti-rPru $p 3$ IgE values, however, the latter were clearer indicators of patients at major risk of severe reactions, given that anti-peach $\operatorname{IgE}$ values did not reach a similar positive cutoff.

Concerning pollen sensitisation we observed that patients with mild OAS, as compared to patients with severe OAS, reacted with greater frequency to all the main pollens both in terms of symptoms and specific IgE levels, with the exception of mugwort which showed a frequency of sensitisation that was similar between the two groups. Moreover, in the group of patients with severe OAS, mugwort was the highest sensitising pollen. An explanation for this finding is that mugwort pollen contains Art v 3, an LTP that cross-react with Pru p 3 [20], and that almost all group B patients were LTP-sensitised even if few of them presented clinical pollinosis.
The final consideration underlines the crucial role played by the availability of peach recombinant allergens in evaluating the IgE sensitization profile in peach-allergic patients. In the present study, we found that positivity to the three peach recombinant allergens was sufficient to diagnose peach allergy in all recruited patients. Furthermore, this study incontrovertibly shows that the pattern of allergen sensitisation clearly influences clinical reactivity, and that IgE positivity to the different allergens conditions symptom severity. Thus showing that component-resolved diagnosis for peach can provide notable advantages in the diagnosis of peach food allergy. Therefore, in conclusion, our results show that the positivity to rPru p 3 gives way to a greater risk of severe peach induced reactions, while positivity to rPru $\mathrm{p} 1$ is associated to a lower risk of severe reactions. Moreover, patients positive to rPru p 3 and simultaneously positive to $\mathrm{rPru} \mathrm{p} 1$ and 4 were conferred a sort of 'protective' effect against the development of severe symptoms induced by antirPru p 3 IgE. This point highlights that the existence of different forms of co-sensitisation could determine clinical situations that are significantly different from those seen in monosensitisation. This novel concept of variable clinical severity as an inverse function of the number and types of peach allergens that one is sensitised to, is clearly evident in this study and has not been previously addressed in the literature. In summary, in Northern Italy the high concentration of birch allows for co-sensitisation to Bet $\mathrm{v} 1$ homologues and LTP, and in these cases the favourable clinical feature is conditioned by sensitisation to birch homologues.

\section{Acknowledgements}

This study has been partially sponsored by Phadia Italia Srl and Sogemi Foods-for Expo Milan 2015.

References

Int Arch Allergy Immunol 2011;156:362-372 
4 Kivity S, Dunner K, Marian Y: The pattern of food hypersensitivity in patients with onset after 10 years of age. Clin Exp Allergy 1994; 24:19-22.

$\checkmark 5$ Ben M'rad S, Dridi A, Douggi MH, Khouani H, Tritar F, Merai S, Djernayah F: Food allergy to peach. Rev Fr Allergol 2005;45:385388.

-6 Rodrigues-Alves R, Lopez A, Pereira-Santos $\mathrm{MC}$, et al: Clinical anamnestic and serological features of peach allergy in Portugal. Int Arch Allergy Immunol 2009;149:65-73.

-7 Fernández-Rivas M, González-Mancebo E, Rodríguez-Pérez R, Benito C, SánchezMonge R, Salcedo G, et al: Clinically relevant peach allergy is related to peach lipid transfer protein, Pru p 3, in the Spanish population. J Allergy Clin Immunol 2003;112:789-795.

$\checkmark 8$ Asero R, Antonicelli L, Arena A, et al: EpidemAAITO: Features of food allergy in Italian adults attending allergy clinics: a multi-centre study. Clin Exp Allergy 2009;39:547-555.

9 Asero R, Antonicelli L, Arena A, et al: Causes of food-induced anaphylaxis in Italian adults: a multi-centre study. Int Arch Allergy Immunol 2009;150:271-277.

-10 Ebner C, Hirschwehr R, Bauer L, Breiteneder $\mathrm{H}$, Valenta R, Ebner H, et al: Identification of allergens in fruits and vegetables: IgE crossreactivities with the important birch pollen allergens Bet v1 and Bet v 2 (birch profilin). J Allergy Clin Immunol 1995;95:962-969.
1 Pastorello EA, Farioli L, Pravettoni V, Ortolani C, Ispano M, Monza M, et al: The major allergen of peach (Prunus persica) is a lipid transfer protein. J Allergy Clin Immunol 1999; 103:520-526.

12 Gamboa PM, Caceres O, Antepara I, Sanchez-Monge R, Ahrazem O, Salcedo G, et al: Two different profiles of peach allergy in the north of Spain. Allergy 2007;62:408-414.

13 Pastorello EA, Pravettoni V, Farioli L, Ispano M, Fortunato D, Monza M, et al: Clinical role of a lipid transfer protein that acts as a new apple-specific allergen. J Allergy Clin Immunol 1999;104:1099-1106.

14 Sampson HA, Munoz-Furlong A, Cambell $\mathrm{RL}$, et al: Second symposium on the definition and management of anaphylaxis: summary report. Second national institute of Allergy and Infectious Disease/Food Allergy and Anaphylaxis Network symposium. J Allergy Clin Immunol 2006;117:391397.

15 Bindslev-Jensen C, Ballmer-Weber BK, Bengtsson U, Blanco C, Ebner C, Hourihane J, Knulst AC, Moneret-Vautrin DA, Nekam K, Niggemann1 B, Osterballe M, Ortolani C, Ring J, Schnopp C, Werfel T: Standardization of food challenges in patients with immediate reactions to foods - position paper from the European Academy of Allergology and Clinical Immunology. Allergy 2004;59: 690-697.
16 Dreborg S: Skin Tests used in type 1 allergy testing: Position paper of the European Academy and Clinical Immunology. Allergy 1989;44:22-59.

$>17$ Fernandez-Rivas M, Bollhaar S, GonzalezMancebo E, Asero R, van Leeuwen A, Bohle B, et al: Apple allergy across Europe: how allergen sensitization profiles determinate the clinical expression of allergies to plant foods. J Allergy Clin Immunol 2006;108:481-488.

18 Johnson DA, Barton GJ: Mast cell tryptases: examination of unusual characteristics by multiple sequence alignment and molecular modelling. Protein Science 1992;1:370-377.

19 Wijesinha-Bettoni R, Alexeev Y, Johnson P, et al: The structural characteristics of nonspecific lipid transfer proteins explain their resistance to gastroduodenal proteolysis. Biochemistry 2010;49:2130-2139.

20 Pastorello EA, Pravettoni V, Farioli L, Rivolta F, Conti A, Ispano M, et al: Hypersensitivity to mugwort (Artemisia vulgaris) in patients with peach allergy is due to a common lipid transfer protein allergen and is often without clinical expression. J Allergy Clin Immunol 2002;110:310-317. 